\title{
ICON and the patient's perceptions of malocclusion
}

\author{
The relationship between Index of Complexity, Outcome and Need, and patients' perceptions of malocclusion: a study \\ in general dental practice by A-R Koochek, M. Shue-Te Yeh, B. Rolfe and S. Richmond Br Dent J 2001; 191:325-329
}

\begin{abstract}
Aim
To examine the relationship between the Index of Complexity, Outcome and Need (ICON) and the subjective opinions of patients attending for routine dental care.
\end{abstract}

\section{Materials and methods}

This study was undertaken at two general dental practices in Cardiff and Bedford. 50 patients aged between 11-14 years and 50 patients aged $30-40$ years presenting for routine dental treatment were selected in each. The subjects were assessed objectively using the ICON guidelines by two examiners trained and calibrated in the use of this index. The scores were recorded directly from the patient. Subjective assessments were obtained from the patients by means of a questionnaire consisting of four simple questions addressing aesthetics, function, speech and treatment need using a five point Likert scale.

\section{Results}

The mean ICON scores for the different genders and age groups participating in this study were; $11-14$ year old males 58.4 (SE 3.17 ); 11-14 year old females 51.8 (SE 3.51); 30-40 year old males 51.2 (SE 2.70); 30-40 year old females 45.3 (SE 2.56). There were statistically significant differences in ICON scores between the younger and older groups $(P=0.024)$ and females and males $(P=0.04)$. Adult patients were more likely to reject treatment than younger patients. Analysis of the professional scores in relation to subjective assessments, using the Spearman rank correlation coefficient, for 11-14 and 30-40 year olds, and for the male and female genders, revealed that the ICON has a significant correlation with patients' perceptions of aesthetics, function, speech and treatment need $\left(r^{2}=0.01\right.$ to 0.28$)$. The only exceptions were patients' perceptions of speech in the 30-40 year old group, and function in the female gender, which did not show a statistically significant correlation to the professional assessments.

\section{Conclusion}

In this study, the ICON was found to correlate with patients' opinions of aesthetics, function, speech and treatment need. The strength of association, however, was low. It can be concluded that the ICON alone is not necessarily a suitable predictor for appearance, function, speech or treatment need for those individuals attending general dental practice for routine dental care. In combination with a simple question to assess the patient's desire for treatment, the shared decision for any particular individual to enter the treatment process can be determined.

In Brief

- Malocclusion is a continuum ranging from ideal occlusion to considerable deviation from normal

- Severity of malocclusion (how far a malocclusion deviates from normal) can be measured objectively using occlusal indices

- Demand for orthodontic treatment results from an individuals desire to seek treatment because of a perceived problem with the dentition and/or surrounding structures

- The need and demand for orthodontic treatment can fluctuate through the individuals life caused by 'life cycle events' including change in wealth and social conditions

- Need and demand are also influenced by dental development, facial growth, facial changes and social awareness.

\section{Comment}

7 his paper attempts to evaluate the use1 fulness of an index of complexity outcome and treatment need (ICON). The limitations of the PAR and IOTN have been recognised for some time but they are still used to try to evaluate the results of treatment. As they do not take into account the multifactorial nature of malocclusion and the degree of improvement aimed for by the orthodontist, the ICON has been developed and tested both in the UK and abroad by various orthodontists. The index takes into account five components: the aesthetics, upper arch crowding/spacing, crossbite, incisor open bite/overbite and buccal segment anterior posterior relationship.

This study was designed to determine whether there was a relationship between patient's perception of their malocclusion and the ICON. The groups were carefully selected and this selection excluded the handicapped and all those who had or wanted orthodontic treatment leaving a very limited sample that presumably had very little knowledge of malocclusion and its problems. If the patients desiring orthodontic treatment had been included it would have produced an indication of the value of the ICON for routine orthodontic patients. Also the position of the general practices, whether from a working class area or from the more affluent middle class area, might also influence the response of the patients.

The last question in the questionnaire given to the patients was worded in a way that suggested that they needed treatment and this may have biased the answers. If this had been worded differently maybe the results would have been different.

The ICON was found to have a significant correlation with patient's perceptions of aesthetics, function, speech and treatment need but the strength of correlation was low and indicated that the ICON is not necessarily a suitable predictor for aesthet- ics, function, speech and treatment need. Adult patients are more likely to decline treatment than younger patients.

The ICON was good at detecting those patients that required treatment but did not reject those patients that did not need orthodontic treatment. It was difficult to predict the patient's attitude to the arrangement of their teeth and their perception of need using a single index.

The ICON attempts to incorporate the patient's and clinician's perceptions of orthodontic treatment into a single index which is important for the patient who needs to get satisfaction from the treatment and the clinicians who assess the needs and set the standards. This study has not resulted in conclusive evidence for the index in this carefully selected group of patients.

\section{J. P. Moss}

Honorary Consultant, St Bartholomew's and the Royal London Hospital 